\title{
Padrões de Uso da Tecnologia Digital no Aprendizado de um Curso de Medicina
}

\author{
Title: Patterns of Technologies' Use in the Learning at a Medicine Course
}

Giovanna Truyts Biscardi

Faculdade de Medicina de São José do Rio Preto

gtbiscardi@outlook.com

\author{
João Marcelo Rondina \\ Faculdade de Medicina de São José do Rio Preto \\ Serviço Nacional de Aprendizagem Comercial \\ rondina@famerp.br
}

\begin{abstract}
Resumo
A Geração Y é aquela que nasceu sob a influência das tecnologias digitais. Atualmente, essa população é representada pelos jovens universitários que criaram um vínculo estreito e rotineiro com a Internet e suas ferramentas. Pesquisas afirmam que essa geração de jovens imediatistas e habituados à multitarefa desenvolveu um novo tipo de neuroplasticidade e, portanto, possuiriam hábitos e mecanismos de aprendizado peculiares que deveriam ser respeitados e considerados na dinâmica de ensino e aprendizagem. Entretanto, existem poucos estudos avaliando a real existência dessas características nos jovens universitários. Dessa forma, apresentamos as conclusões do estudo descritivo com abordagem qualitativa realizado com estudantes de Medicina da Faculdade de Medicina de São José do Rio Preto que visa conhecer como os universitários usam as tecnologias digitais e o impacto de seus hábitos e comportamentos quanto ao envolvimento dessas novas tecnologias em seus estudos. Percebeu-se que a tecnologia exerce importante influência na vida dos estudantes, porém continua sendo uma entre várias outras, o que faz com que o professor mantenha um papel fundamental na formação desses alunos considerados nativos digitais, principalmente ajudando e guiando-os na utilização correta e completa das novas tecnologias desenvolvidas para o aprendizado
\end{abstract}

Palavras-Chave: Internet; Aprendizagem; Estudantes de Medicina; Grupos Populacionais; Educação Médica

\begin{abstract}
The Y generation is understood as that born under the influence of the digital technologies. Now it's represented by the young university students that created such a tight bond with the internet and its tools that uses them easily and frequently. Many works say that this generation of immediate young people accustomed to multitasking has developed a new kind of neuroplasticity entirely different of their antecessors and therefore possess peculiar habits and mechanisms of learning that should be respected and considered in the dynamics of teaching and learning, creating a pressure in the educational institutions to adequate in this reality. However, there are a few studies evaluating the real existence of these characteristics. Thus, we preset the conclusions of this descriptive qualitative project fulfilled with medicine students at Faculdade de Medicina de São José Rio Preto that aim to know how these students use digital technologies and the impact of their habits and behaviors regarding the involvement with technologies in their studies. The conclusion was that technologies are an important influence on student's lifes, however continues to be one among several others, which allows the teachers to keep a fundamental role in the formation of these digital natives, especially helping and guiding them in the correct and full use of new technologies developed for learning.
\end{abstract}

Keywords: Internet; Learning; Medical Students; Population Groups; Medical Education

Cite as: Biscardi, G. T., \& Rondina, J. M. (2017). Patterns of Technologies' Use in the Learning at a Medicine Course (Padrões de Uso da Tecnologia Digital no Aprendizado de um Curso de Medicina). Brazilian Journal of Computers in Education (Revista Brasileira de Informática na Educação - RBIE), 25(1), 01-15. DOI: 10.5753/RBIE.2017.25.01.1 


\section{Introdução}

A geração que está frequentando o Ensino Superior é conhecida como Geração Y, Geração Net (Ebner \& Schönm, 2012), Geração Eu (Twenge, 2009) ou como Nativos Digitais (Jones, 2012). A Geração Y englobaria os estudantes universitários entre 18 e 35 anos (Rennie \& Morrison, 2013) que cresceram sob as influências do avanço tecnológico digital e da Internet na chamada Era da Informação. São indivíduos que estão sempre conectados através de seus notebooks, tablets e smartphones - dentro ou fora das salas de aula - comunicando-se com outras pessoas e interagindo constantemente com uma vasta gama de conteúdos (Lombardía, 2008) (Oliveira, 2015).

Existe uma grande discussão na comunidade científica a respeito da Geração Y no que tange suas características, prioridades, modo como eles aprendem e deveriam ser ensinados. Todavia, existem pesquisas indagando se esta população realmente existe de fato e quais as características elencadas nos estudos científicos se fazem presentes nestes indivíduos (Jones \& Healing, 2010).

A literatura refere-se à Geração Y como aqueles nascidos após 1980, na consolidação das tecnologias digitais. Esses indivíduos teriam acesso à Internet e habilidade suficiente para utilizarem seus serviços (Jones, 2012) e serem capazes de dominar essas mídias como as gerações anteriores jamais conseguirão. $\mathrm{O}$ contato íntimo com a tecnologia explicaria a natureza multitarefa e impaciente desta geração, além de outras características como necessidade de acesso rápido a informações, de resultados e recompensas imediatas, baixa tolerância a atrasos na comunicação, preferência para construção ativa de conhecimento e de ambientes interativos (Ebner \& Schönm, 2012).

Alguns autores defendem que este público desenvolveu mais o hemisfério direito do cérebro devido aos estímulos da Internet e dos jogos eletrônicos (contrastando com o uso do hemisfério esquerdo em atividades de leitura, por exemplo) (Lombardía, 2008) (Oliveira, 2015) e por isso necessitariam de métodos de aprendizagem diferenciados e que, portanto, o tradicional modo de ensino não seria suficiente (Prober, 2012) (Conole \& Alevizou, 2010). Ressalta-se que tais suposições sobre a neuroplasticidade desta geração carecem de mais evidências comprovadas cientificamente (Thompson, 2013).

Como resultado desse pensamento orgânico diferente, é de se esperar o surgimento de estudos sobre a Geração Y no ambiente educacional. A maioria das novas estratégias de ensinoaprendizagem tem em seu cerne o uso da tecnologia, principalmente as ferramentas da Web 2.0. A utilização deste tipo de mídia tem facilitado a criação de novos espaços de construção de conhecimentos, permitido alargar o tempo e espaço em que as aprendizagens podem ocorrer.

O impacto das inovações tecnológicas na sociedade tem contribuído para a criação de novas estratégias de ensino e aprendizagem, bem como na capacitação de seus educadores (Torres \& Amaral, 2011). Já se verificou, por exemplo, a viabilidade do uso do Facebook enquanto ambiente informal de aprendizagem com a presença de professores convidados a explicar a aplicação da teoria estudada (Cain \& Policastri, 2011). Em outras instituições, observou-se como as tecnologias viriam a customizar as aulas e otimizar o tempo dos estudantes em um fenômeno denominado flipped classroom. Nesse modelo, as mídias digitais ficam a cargo de transmitir a informação propriamente dita enquanto o professor alimenta discussões e instiga o pensamento crítico do estudante em casos clínicos e simulações no curso de Medicina. Parte desse interesse pelas instituições de ensino nas tecnologias se dá pelo fato de serem fáceis de criar, oferecerem flexibilidade de horário para que os estudantes aprendam em seu próprio ritmo, além de serem populares e passíveis de grande adesão pelos mesmos (Prober, 2012). 
A adesão da tecnologia na sala de aula não se insere somente nos métodos alternativos de ensino médico (Castanha \& Castro, 2010). No método tradicional, muitos professores estimulam os estudantes a serem autores criativos de conteúdo, produzindo e manipulando imagens de vídeo (disponibilizando-as depois em serviços como o YouTube), usando palavras-chave (Tags) para criar taxonomias que tornam mais fácil e eficaz a procura de informação em blogs, ou participando dinâmica e coletivamente na construção de enciclopédias virtuais como a Wikipedia (Jimoyiannis \& Angelaina, 2012). Ou ainda utilizam programas como Homem Virtual, software de animação desenvolvido pelo Departamento de Patologia - equipe de Telemedicina na Universidade de São Paulo (USP) (USP, 2016), e sites de animações como o portal de vídeo aulas de Embriologia da Universidade Federal Fluminense (Santos \& Carvalho, 2009) como ferramentas para auxiliar no ensino de conteúdos densos como Embriologia ou Anatomia, típicas do ciclo básico médico.

A importância e relevância do tema motivam a realização desta pesquisa, visto que a investigação do perfil dos estudantes em relação às tecnologias digitais poderá permitir que a instituição e os professores planejem melhor a estrutura tecnológica e informacional oferecida aos estudantes. Isso, pois a tecnologia não deve ser implementada demasiadamente, como se observa na maioria das vezes, mas com eficácia. Vale ressaltar que estes dados também poderão ser usados em pesquisas sobre e-learning, andragogia, ensino médico, entre outros.

\section{Materiais e Métodos}

Foi realizada uma pesquisa do tipo descritivo, como fonte de coleta de dados primeiramente foi feita a pesquisa bibliográfica, na forma de revisão na literatura e posteriormente foi aplicado o questionário elaborado pela autora, baseando-se no publicado por Thompson (Thompson, 2013). Esse estudo foi realizado com o apoio do Programa Institucional de Bolsas de Iniciação Científica da Faculdade de Medicina de São José do Rio Preto (PIBIC/FAMERP) com número de Certificado de Apresentação e para Apreciação Ética (CAAE) 32771214.6.0000.5415, no período de agosto de 2014 a julho de 2015 .

Os dados do tipo bibliográfico foram, preferencialmente, artigos publicados em periódicos e eventos científicos, nacionais e internacionais. Para a realização desta pesquisa, foram selecionados artigos que abordam os principais aspectos relacionados à educação médica com uso das tecnologias digitais.

O levantamento realizado em campo envolveu um questionário impresso com perguntas respondidas em escalas de 5 pontos, acompanhado de um Termo de Consentimento Livre e Esclarecido (TCLE). Esse questionário foi respondido de forma voluntária, após a assinatura do TCLE, pelos estudantes do curso de graduação em Medicina da FAMERP. Os dados do estudo foram armazenados numa planilha eletrônica do software MS - Microsoft Excel Versão 2013. $\mathrm{Na}$ análise dos dados utilizou-se estatística descritiva simples, calculando frequência absoluta, relativa e o desvio padrão das amostras.

$\mathrm{O}$ instrumento de coleta foi um questionário desenvolvido pela autora, baseando-se no aplicado originalmente online por Thompson em calouros de uma universidade do Centro-Oeste americano que tinha por objetivo conhecer como os universitários usam as tecnologias digitais e o impacto de seus hábitos e comportamentos quanto ao envolvimento da tecnologia em seus estudos.

O questionário aplicado pela autora foi estruturado em três partes:

- Parte 1: Informações demográficas básicas. Os participantes são questionados sobre sua idade, sexo escolaridade. 
- Parte 2: Escala do perfil digital e dos hábitos eficazes de aprendizagem. Os participantes, de acordo com sua opinião retirada de perguntas avaliadas em escala de 5 pontos, são classificados como estudantes mais "tradicionais" ou mais "digitais" e classificam quais dos seus comportamentos são mais ou menos produtivos para o estudo.

- Parte 3: Escala do uso da tecnologia. Os participantes classificam a frequência com que usam 19 das principais ferramentas tecnológicas.

Os critérios de inclusão na população analisada foram: o participante deverá ser estudante do curso de Medicina da FAMERP, englobando todas as séries que ingressaram na faculdade entre os anos de 2010 e 2015 (englobando as turmas XLIII, XLIV, XLV, XLVI, XLVII, XLVIII), do sexo masculino ou feminino, com idade entre 18 e 35 anos. A população analisada teve $\mathrm{N}$ de 78 elementos caracterizando uma amostra aleatória por conveniência, sendo distribuídos de acordo com os tamanhos das turmas.

Os dados adquiridos no questionário foram obtidos a partir de variáveis qualitativas. No processo de análise e interpretação dos dados foram utilizados diversos procedimentos: codificação das respostas, tabulação dos dados e cálculos estatísticos. Os procedimentos referentes aos cálculos estatísticos têm como objetivo permitir construção de enunciados descritivos sobre alguma população, isto é, descobrir a distribuição de certos traços e atributos (Prodanov \& Freitas, 2013).

\section{Resultados}

Os resultados serão apresentados conforme a estrutura do questionário, ou seja, divididos em três partes. A primeira parte indagava ao participante sobre suas informações demográficas básicas e sobre os aparelhos eletrônicos que possuem. A média das idades dos 78 alunos entrevistados foi de 21,4 anos.

Os participantes foram indagados, também, em relação ao sexo, ao estado civil e ao estado de origem. Dentre os 78 alunos entrevistados, 35 são do sexo masculino e 43 do sexo feminino, sendo que apenas um deles é divorciado, os outros 77 são solteiros. São Paulo é o estado de origem da maioria dos alunos, com $87 \%$ de frequência relativa.

Mais uma pergunta feita para os alunos foi o tempo semanal que eles consideram que tem disponível para a realização de atividades acadêmicas extracurriculares, a frequência das respostas pode ser observada na Tabela 1.

Tabela 1: Tempo semanal disponível para atividades extracurricu
\begin{tabular}{|c|c|c|}
\hline Tempo & $\begin{array}{c}\text { Frequência } \\
\text { Relativa }\end{array}$ & $\begin{array}{c}\text { Frequência } \\
\text { Absoluta }\end{array}$ \\
\hline 1-3 horas & $19 \%$ & 15 \\
\hline 3-5 horas & $23 \%$ & 18 \\
\hline $5-8$ horas & $26 \%$ & 20 \\
\hline 8-10 horas & $17 \%$ & 13 \\
\hline Mais 10 horas & $15 \%$ & 12 \\
\hline
\end{tabular}

Quanto ao acesso às tecnologias, a pergunta permitia mais de uma resposta, sendo que o participante marcava quais possuía, eram citadas: Smartphone, Computador, Notebook, Tablet e Outros (permitia o preenchimento manual). As porcentagens encontradas encontram-se no Gráfico 1. A pergunta foi abrangente em relação às principais tecnologias, pois apenas um aluno preencheu a opção outros, com videogame. 


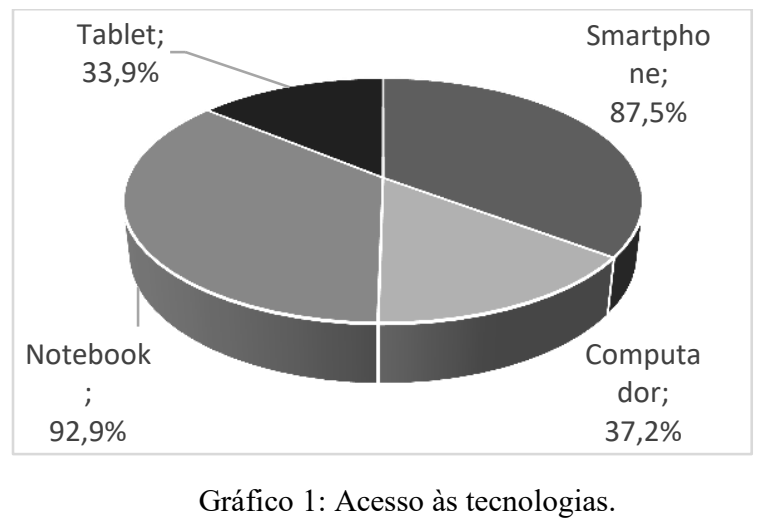

\subsection{Aspectos do Perfil Digital dos Estudantes}

A segunda parte dos dados é referente às perguntas sobre o perfil digital e os hábitos eficazes de aprendizagem. A primeira análise feita diz respeito à preferência dos alunos por materiais impressos ou digitais, a frequência das respostas na escala de 5 pontos pode ser vista no Gráfico 2. As respostas variavam entre "devagar e focado ponto a ponto", número 1, "rápido, explorando o todo", número 5.

Outra indagação feita foi em relação ao hábito de realizar múltiplas tarefas simultaneamente, ou seja, se o aluno enquanto estuda fica checando o celular ou vendo televisão, dentre outros. A resposta dessa questão variava de "foca em uma coisa por ver", número 1, até "faz coisas simultâneas constantemente", número 5; os dados coletados podem ser observados no Gráfico 3, na primeira coluna.

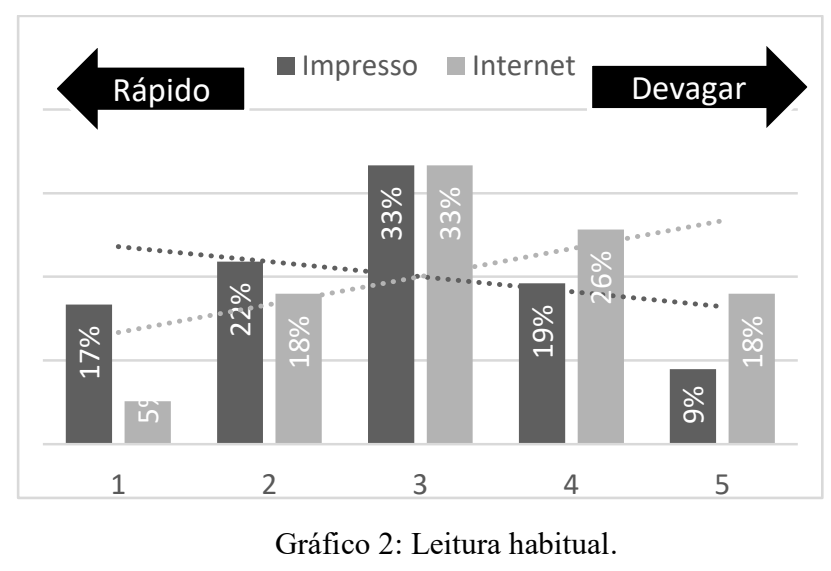

Além de analisar se o aluno é capaz e faz atividades simultâneas, ele foi indagado se essas atividades estavam ou não relacionadas, a escala dessa questão variava de "faz tarefas não relacionadas simultaneamente", número 1, até "faz tarefas relacionadas simultaneamente", número 5. Aproximadamente $15 \%$ dos alunos declararam que não realizam tarefas simultaneamente. 


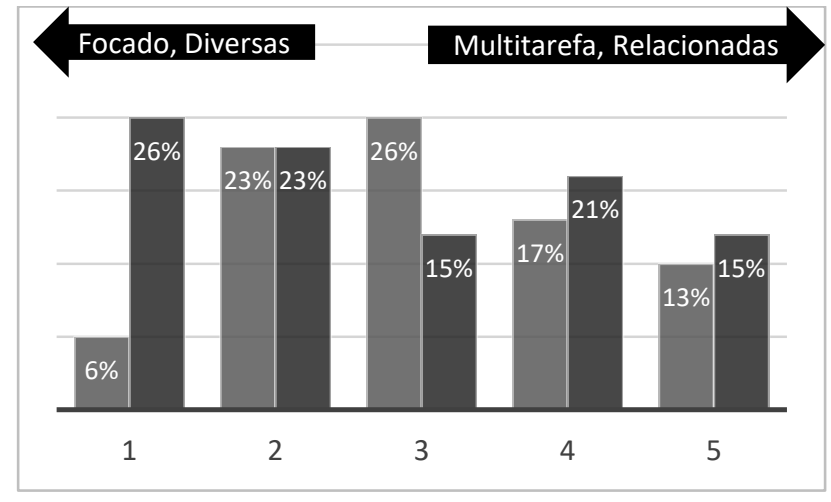

Gráfico 3: Hábito de realizar multitarefas, relacionadas ou não.

Já o Gráfico 4, apresenta as frequências relacionadas aos alunos focarem mais nos textos ou nos gráficos do material em que estão estudando.

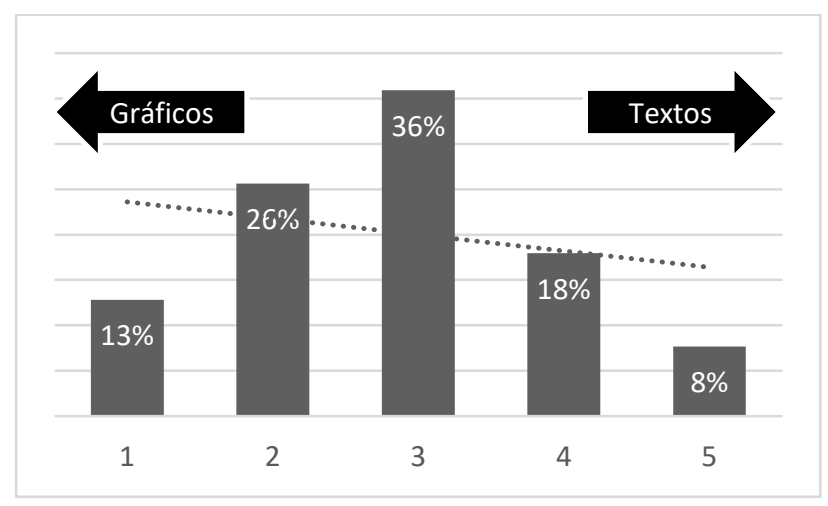

Gráfico 4: Aprendizado mais focado em gráficos ou textos.

Uma diferenciação importante realizada foi entre as dúvidas e/ou interesses correlacionados que surgiam no estudo de materiais digitais ou impressos. Nessas perguntas os alunos poderiam responder variações entre "lê a página inteira antes de clicar nos links" ou "anota a pergunta para procurar a resposta mais tarde", número 1, e "clica em todos os links assim que os vê" ou "procura a resposta imediatamente", número 5. Os dados são apresentados no Gráfico 5.

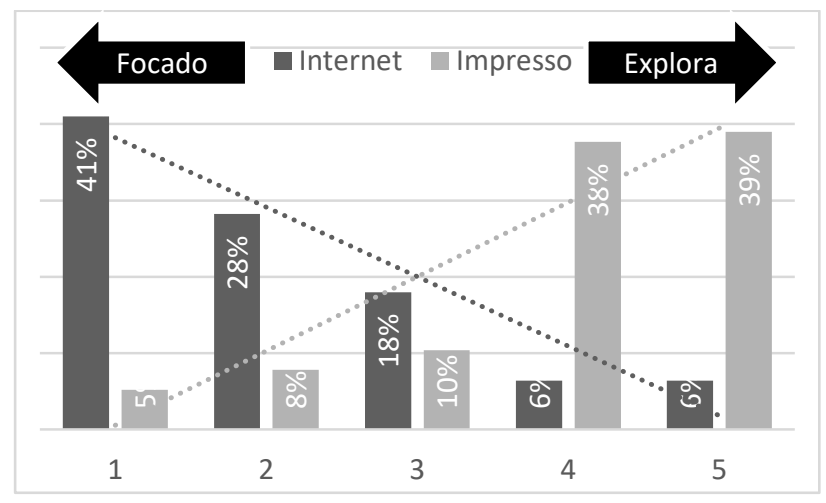

Gráfico 5: Dúvidas ou interesse correlacionado.

As próximas perguntas do questionário estavam relacionadas à habilidade do aluno de se concentrar em aprender algo e se comunicar ou trabalhar com amigos simultaneamente. $\mathrm{O}$ Gráfico 6 mostra a comparação entre os alunos se comunicarem com os amigos enquanto estudam, seja cara-a-cara, online ou por mensagens, e as preferências de estudar sozinho ou acompanhado desses amigos. As respostas possíveis para essas perguntas eram "sem contato" e "sozinho", número 1, e "contato constante" e "com amigos", número 5. 


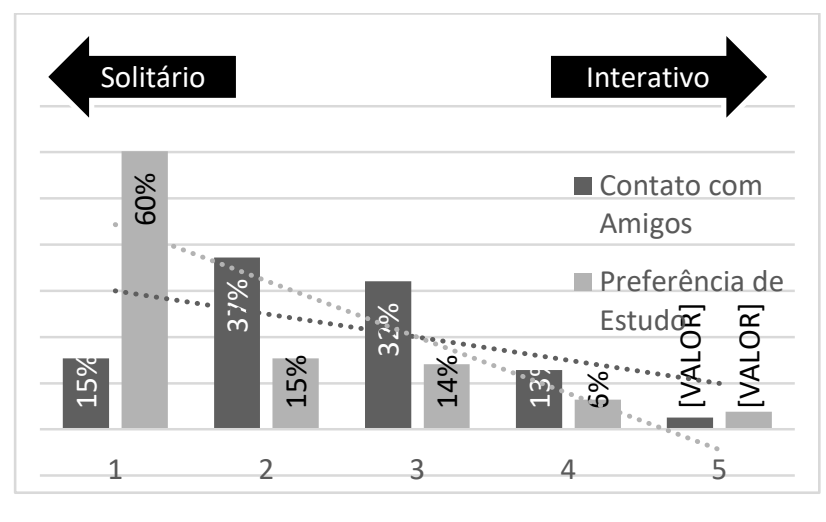

Gráfico 6: Interatividade durante trabalhos em grupo.

Outra abordagem feita teve relação com o funcionamento de trabalhos em grupo, para tanto os alunos foram indagados na forma como dividiam o tempo entre socializar e trabalhar. As respostas possíveis variavam de "passa a maior parte do tempo socializando", com $23 \%$ dos participantes, até "passa a maior parte do tempo trabalhando na atividade", com 15\%. Entretanto, 59\% dos participantes relataram que a interatividade durante o estudo divide-se entre a socialização e o trabalho, e 3\% afirmou que não estuda ou faz trabalhos com seus colegas.

A seguir, o conjunto de perguntas proposto foi para estudar a eficácias dos atuais métodos de ensino, por exemplo sala de aula na qual o aluno é apenas o receptor da informação, em comparação com métodos menos tradicionais, por exemplo realização de projetos nos quais o aluno participe ativamente na construção do seu conhecimento; a preferência dos alunos por cada um desses métodos está apresentada no Gráfico 7, as respostas variavam de "ter a informação passada para mim", número 1, até "encontrar a informação por conta própria", número 5.

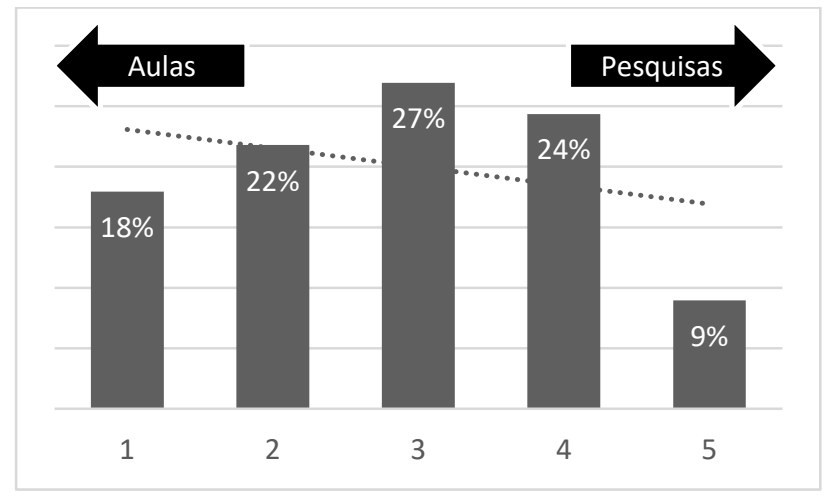

Gráfico 7: Preferências no aprendizado.

O Gráfico 8 mostra dados em que os alunos classificaram a experiência de assistir uma aula; as respostas variavam de "entediado, ansioso ou sonhando acordado na maior parte do tempo", número 1, até "atento e disposto a aprender", número 5. Já o Gráfico 9 faz uma análise similar ao 8 porém envolvendo o trabalho ativo em um projeto, as respostas variavam de "foca tanto na atividade que não tem tempo para pensar no que deveria estar aprendendo", número 1, até "usa a atividade como um modo de refletir no que está aprendendo", número 5. 


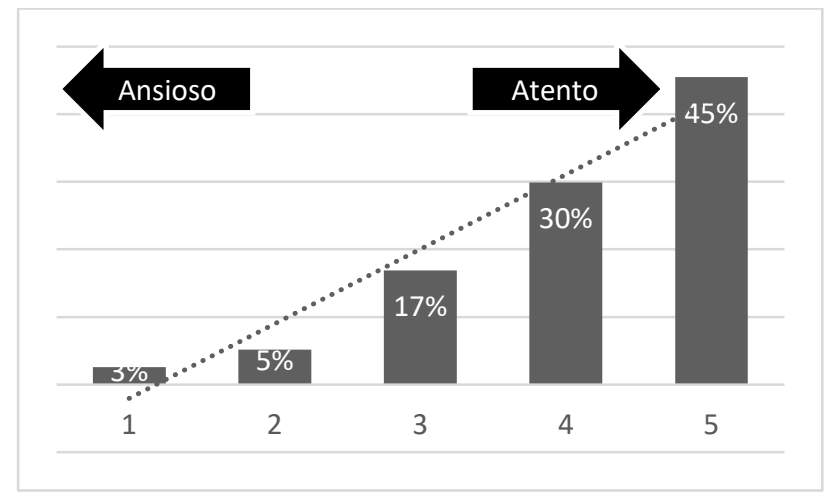

Gráfico 8: Experiência de assistir aula.

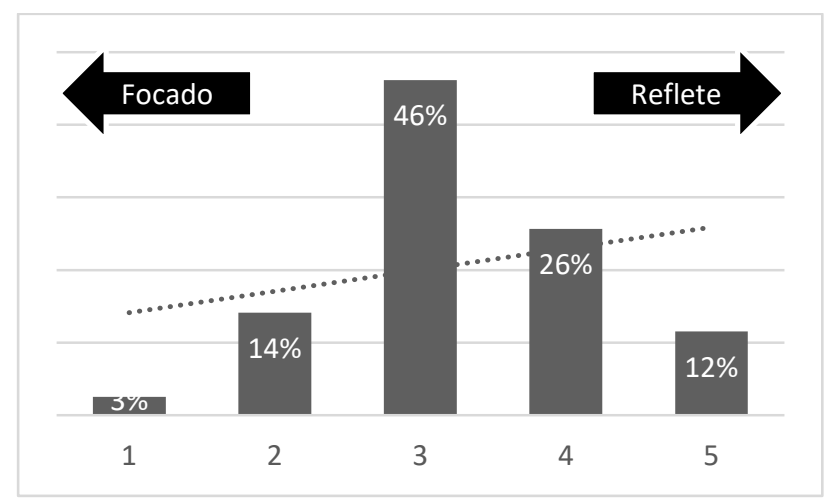

Gráfico 9: Experiência de realizar um projeto.

A última pergunta desse bloco, representada no Gráfico 10, era relacionada ao tédio de curta duração durante os estudos, nos quais sabe-se que a recompensa virá mais tarde, os alunos o classificaram desde "parte normal do aprendizado que eu aceito", número 1, até "intolerável, eu realmente preciso de uma recompensa a cada esforço meu”, número 5.

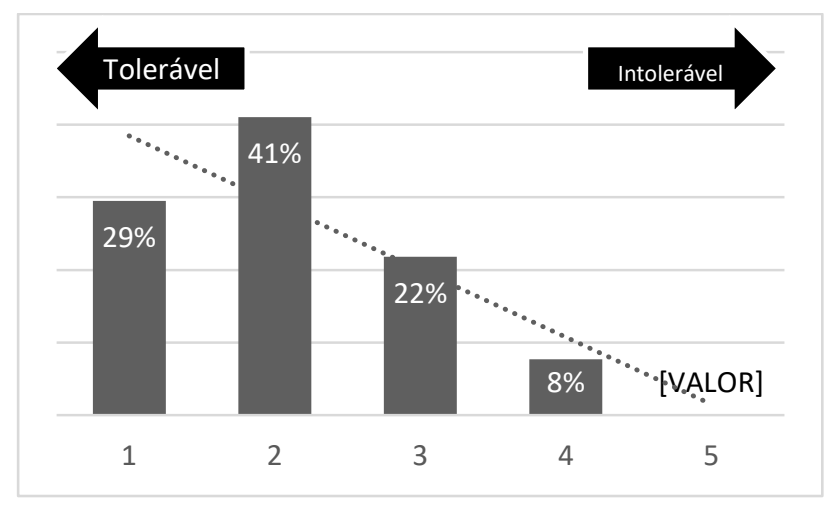

Gráfico 10: Experiência com o tédio originado do aprendizado.

Para finalizar a segunda parte do questionário, os alunos foram indagados sobre o auxílio das tecnologias no aprendizado. A primeira pergunta era a respeito da relação entre trabalho e lazer, os participantes respondiam desde "assumem partes separadas da minha vida", número 1, até "completamente misturadas, número 5; as respostas são apresentadas no Gráfico 11. 


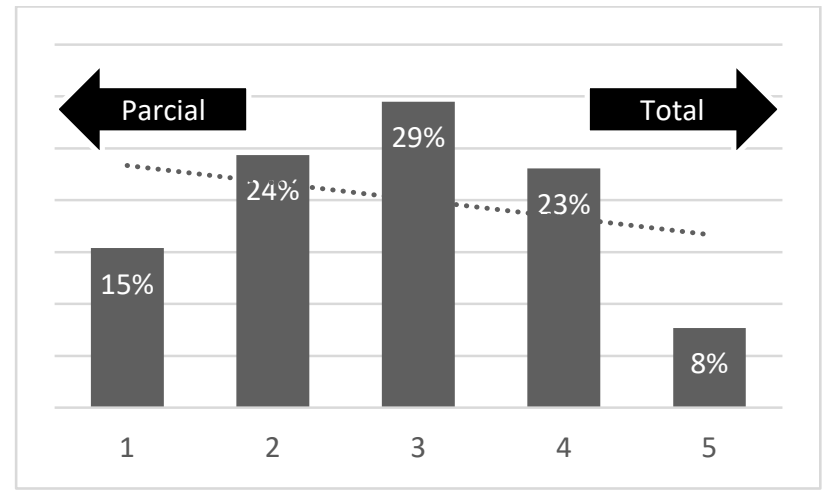

Gráfico 11: Relação entre trabalho e lazer.

A próxima teve relação entre a presença de jogos para estimular o aprendizado, era possível classificar entre "desnecessário", número 1, e "necessário para me deixar interessado", número 5; esses dados são mostrados no Gráfico 12.

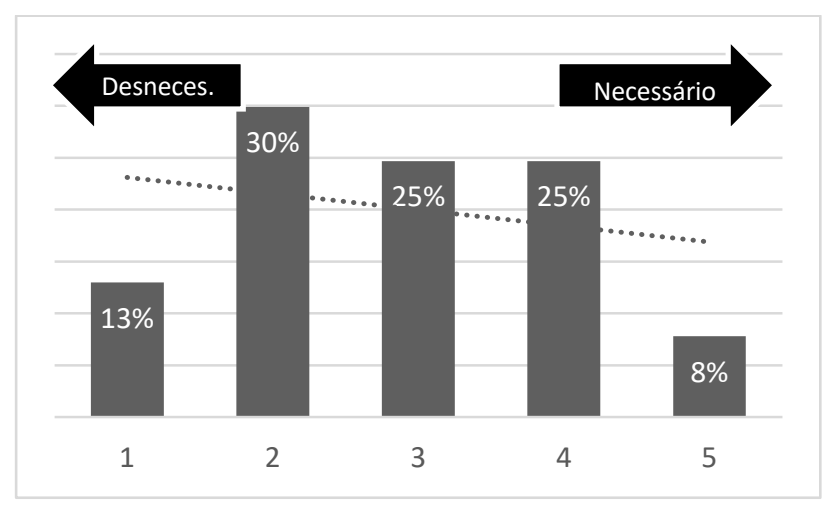

Gráfico 3: Presença de jogos no aprendizado.

Em seguida, foram questionados sobre a importância da tecnologia, podendo ser desde "um elemento desnecessário", número 1, até "faz tanta parte da minha vida que fico desfocado quando não está lá", número 5; os dados colhidos estão no Gráfico 13. Já o Gráfico 14 relata a experiência dos alunos em aprender algo novo utilizando uma tecnologia desconhecida, foi indagado se eles "pensam mais na em si", número 1 , ou se "focam na matéria sem deixar a tecnologia atrapalhar", número 5.

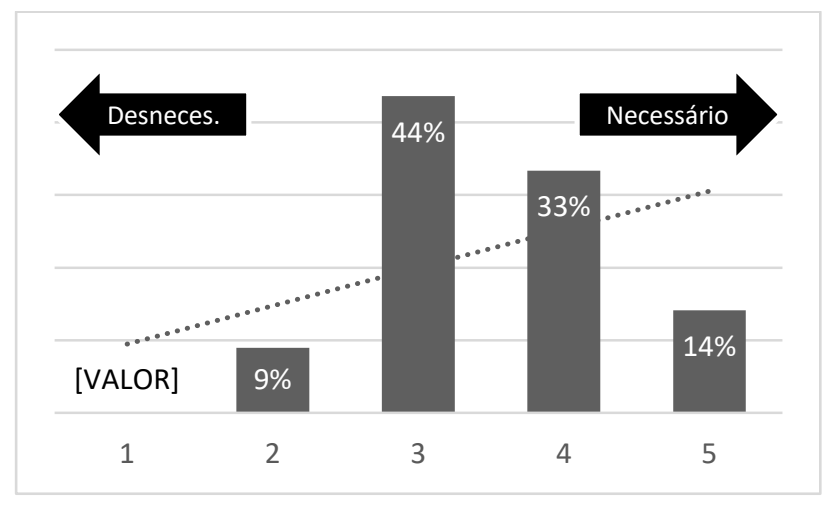

Gráfico 4: Importância da tecnologia no aprendizado. 


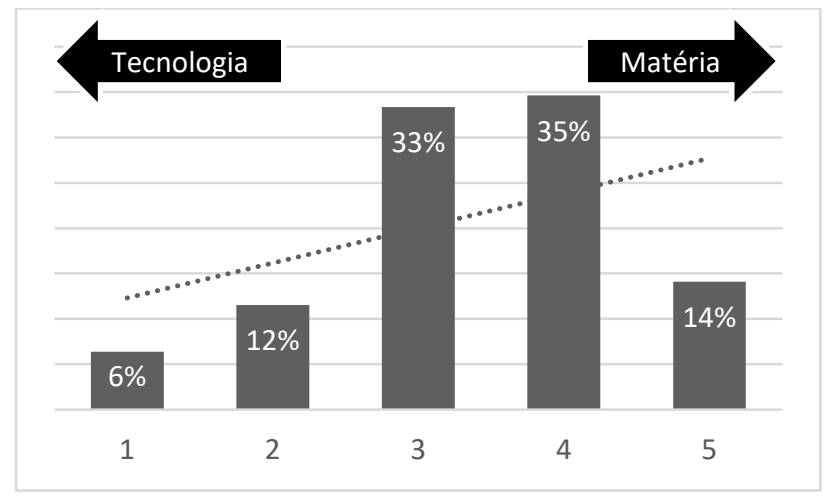

Gráfico 5: Novas tecnologias no aprendizado.

Em relação à importância da tecnologia no aprendizado, $75 \%$ dos estudantes que tem mais de 10 horas disponíveis para atividades extracurriculares consideram-na como um elemento necessário à sua formação. Em comparação com os estudantes que tem menos de 8 horas disponíveis, a avaliação da tecnologia como necessária cai par 57\%.

A terceira parte do questionário era relativa à escala de uso das principais tecnologias disponíveis, os dados são apresentados nos gráficos e tabelas a seguir, o participante respondia a frequência que utiliza tais tecnologias no dia-a-dia, as respostas foram divididas em algumas vezes por dia, por semana, por mês, por ano ou nunca. As primeiras tecnologias avaliadas eram as ferramentas de software, como editor de texto, planilhas de dados e apresentação de slides; os dados são apresentados no Gráfico 15.

Outra ferramenta indagada nessa parte do questionário era a utilização das redes sociais, indicando grande diferença entre o aluno atualizar o próprio perfil e checar por atualizações; os dados coletados estão no Gráfico 16. O Gráfico 17 apresenta as frequências do uso da internet para adquirir conhecimentos, seja lendo um site de conhecimentos ou realizando pesquisas sobre assuntos específicos. Já a Tabela 2 indica todas as porcentagens do uso das outras ferramentas listadas no questionário, inclusive sobre o hábito de realizar multitarefas.

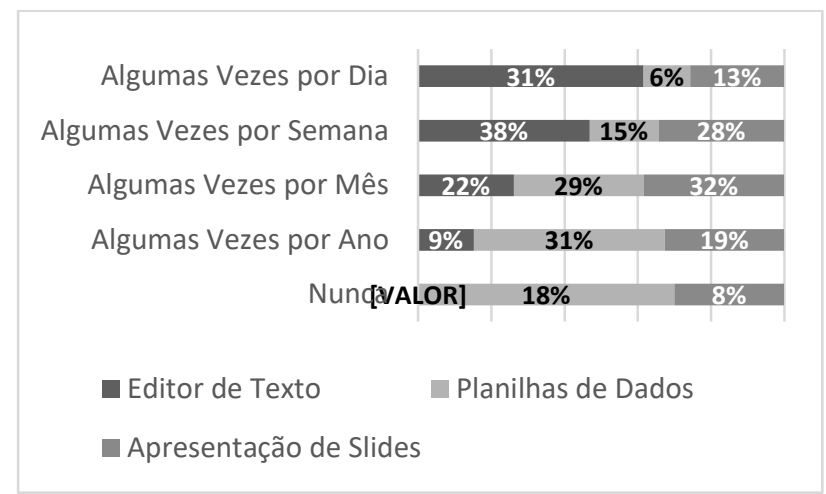

Gráfico 6: Ferramentas de Software. 


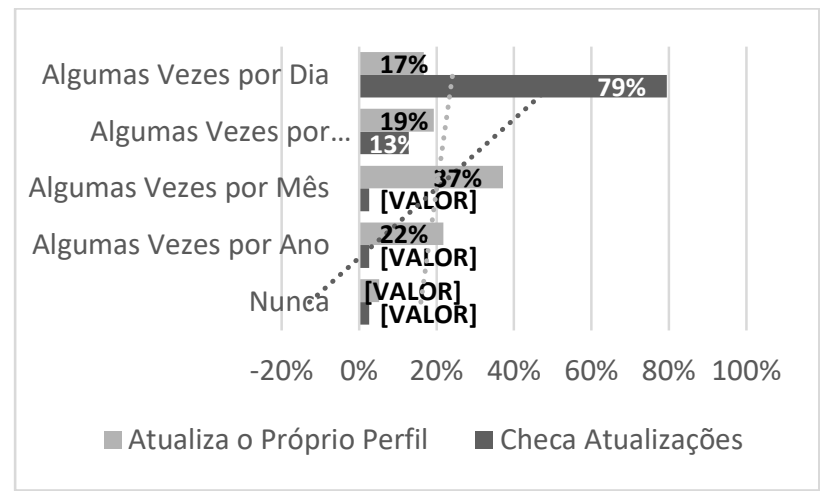

Gráfico 7: Utilização das redes sociais.

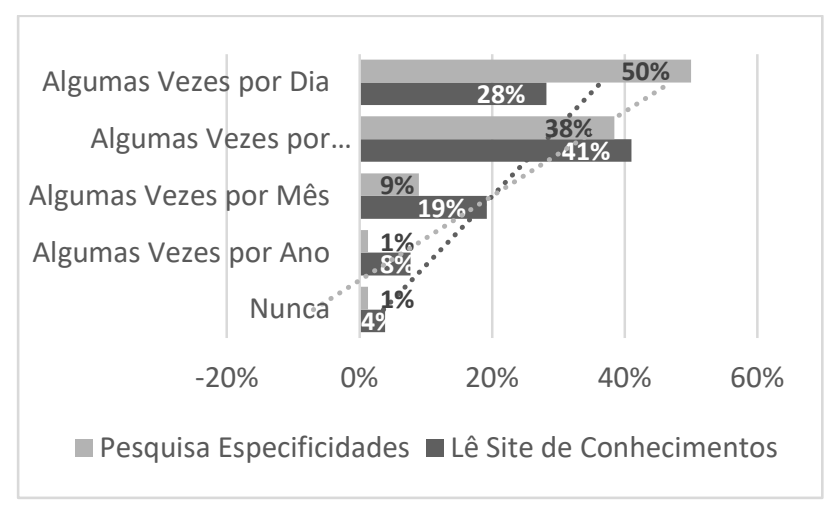

Gráfico 8: Frequência de uso da internet para o aprendizado.

Tabela 2: Frequência de uso das principais tecnologias.

\begin{tabular}{|c|c|c|c|c|c|}
\hline Tecnologia & Nunca & Ano & Mês & Sem. & Dia \\
\hline Compartilhamento de arquivos & $12 \%$ & $6 \%$ & $13 \%$ & $36 \%$ & $33 \%$ \\
\hline Assiste vídeo online & $0 \%$ & $3 \%$ & $15 \%$ & $46 \%$ & $36 \%$ \\
\hline Escuta música online & $0 \%$ & $4 \%$ & $18 \%$ & $42 \%$ & $36 \%$ \\
\hline Cria ou edita vídeo, áudio ou imagem & $24 \%$ & $32 \%$ & $23 \%$ & $12 \%$ & $9 \%$ \\
\hline Cria ou mantém um site & $88 \%$ & $9 \%$ & $0 \%$ & $3 \%$ & $0 \%$ \\
\hline Lê livro & $3 \%$ & $23 \%$ & $28 \%$ & $29 \%$ & $17 \%$ \\
\hline Comenta publicação & $6 \%$ & $8 \%$ & $23 \%$ & $26 \%$ & $37 \%$ \\
\hline Aplicativos de jogos & $22 \%$ & $26 \%$ & $18 \%$ & $12 \%$ & $23 \%$ \\
\hline Jogos online & $53 \%$ & $17 \%$ & $8 \%$ & $6 \%$ & $17 \%$ \\
\hline Mensagens no celular & $0 \%$ & $0 \%$ & $1 \%$ & $5 \%$ & $94 \%$ \\
\hline Chamadas no celular & $0 \%$ & $0 \%$ & $3 \%$ & $18 \%$ & $79 \%$ \\
\hline Uso de duas ou mais tecnologias simultâneas & $9 \%$ & $4 \%$ & $9 \%$ & $32 \%$ & $46 \%$ \\
\hline
\end{tabular}

\section{Discussão}

O propósito deste trabalho especificamente foi analisar as características de aprendizagem da turma de Medicina que pode ser representativa da geração Y, para tal foram analisadas as relações entre as escalas do perfil digital, dos hábitos eficazes de aprendizagem e do uso da tecnologia. 
A segunda parte do questionário foi a mais extensa pois avaliou o perfil digital e os hábitos eficazes de aprendizagem. Com as respostas, foi possível perceber que os alunos consideram que suas leituras são mais rápidas e explorando todo o conteúdo quando possuem materiais impressos em mãos, já quando buscam meios de estudo na internet leem mais devagar, porém mais focados no assusto pesquisado. Além disso, quando estão estudando pela internet, eles tendem a ler primeiro toda a página antes de clicar em qualquer link ou procurar sua dúvida; já quando estão estudando por materiais impressos, eles preferem procurar a resposta em outra fonte imediatamente, ao invés de anotar para procurar a resposta mais tarde. Esses achados são consistentes com a característica da Geração Y de reunir a informação de que precisam de forma rápida e eficiente (Prensky, 2009), o que de certa forma, na visão dos educadores, significa que eles não estão aproveitando ao máximo os conhecimentos disponíveis na web (Kennedy \& Judd, 2011).

Ainda avaliando o perfil digital e os hábitos eficazes de aprendizagem, as respostas encontradas discordam da percepção popular desses alunos necessitarem constantemente de entretenimento (Bauerlein \& Walesh, 2009), já que, durante o estudo, 49\% nos alunos consideram que se mantem mais focados nos estudos, enquanto $15 \%$ conseguem focar mas também realizar multitarefas e $36 \%$ são mais suscetíveis a estudar e realizar outra tarefa simultaneamente. No quesito capacidade de realizar multitarefas, foi avaliado que $15 \%$ dos alunos não as realizam, preferindo focar apenas no estudo ou na diversão, porém os que realizam, normalmente são com atividades não relacionadas, como estudar e escutar música ou usar o celular.

Quando indagados mais a fundo sobre as preferências para se manterem focados nos estudos, os nativos digitais indicaram que preferem analisar gráficos do que textos, porém as respostas foram equilibradas quando indagados sobre a preferência entre assistir uma aula ou realizar um projeto, sendo que $27 \%$, maioria das respostas nessa questão, marcaram a opção do meio na escala de respostas, indicando um balanço entre ter a informação passada ou a adquirida por conta própria. Essa conclusão não se enquadra na concepção das características da Geração Y de Tapscott, que em 1997 foi um dos pioneiros a propor uma lista de 4 comportamentos fundamentais dessa geração, em específico a que afirma uma preferência marcada para a construção ativa do conhecimento juntamente com uma reduzida tolerância a ambientes instrutivos. Outra pergunta relacionada para avaliar a eficácia de cada método de ensino, foi sobre o comportamento desenvolvido pelos estudantes, as respostas informaram que os alunos tendem a ficar atentos e dispostos a aprender durante uma aula de um assusto que consideram importante, mas também conseguem usar atividades, como montagem de seminários, para refletir sobre o conteúdo proposto e não apenas focar em terminá-la rapidamente. Ainda avaliando os métodos de aprendizagem desses alunos, eles responderam sobre o tédio de curto prazo que o aprendizado pode gerar, sendo que $70 \%$ refere que considera tolerável e uma parte integrante do processo de adquirir conhecimentos; isso contraria a percepção popular de que eles estão habituados apenas a interações rápidas e eficazes através dos canais de comunicação (Simões \& Gouveia, 2008).

Um dos principais desafios que essa geração enfrentará ao ingressar no mercado de trabalho é a alta valorização das relações interpessoais (Del Prette \& Del Prette, 2003), nesse contexto os alunos da FAMERP foram indagados sobre a preferência de estudar sozinho ou com colegas e como se relacionam com esses perante a necessidade de trabalhos em grupo. A partir dos dados colhidos, analisou-se que $60 \%$ dos universitários prefere estudar sozinho, apesar de $69 \%$ relatarem que mantém algum tipo de contato com os amigos, seja online ou por mensagens. Quanto à análise do rendimento dos trabalhos em grupo, 48\% referem que passam a maior parte do tempo trabalhando na atividade ao invés de socializar e distrair-se do foco. Outro ponto avaliado nos nativos digitais foi a relação entre trabalho e lazer e a importância de cada um desses fatores na vida deles, as respostas a essa pergunta indicaram que a maioria acredita que 
são relacionados e importantes para a plena realização individual; sendo que as respostam tendem ligeiramente para não estarem totalmente ligados, ou seja, o trabalho pode mas não é o único proporcionador de lazer para a Geração Y. Esses dados podem ser indicativos da eficiência das relações interpessoais dos alunos, mostrando que conseguem socializar e divertirse com atividades em grupo, apresar de não ser o método preferido, e atingir êxito em completar a tarefa proposta.

Finalizando a segunda parte do questionário, os alunos foram indagados sobre o auxílio das tecnologias no aprendizado, pois permitem que o conhecimento seja caracterizado como personalizado, flexível e em permanente construção individual e social (Kenski, 2003). As respostas indicaram que a maioria dos alunos, 47\%, consideram a tecnologia como uma ferramenta necessária para o aprendizado e salientam ainda que mesmo com novas tecnologias conseguem focar mais na matéria a ser aprendida do que na ferramenta. Foram questionados ainda sobre a relevância dos jogos para o aprendizado, os resultados foram bem equilibrados entre os três pontos centrais da escala, porém as respostas tendem a considerar desnecessário, apesar de interessante, a presença dessa ferramenta para tornar o estudo mais atrativo. Esse último dado levantado é oposto à percepção popular de que os integrantes da Geração Y tem grande adesão por jogos (Prensky, 2009), essa conclusão se repete na terceira parte do questionário, quando novamente são indagados sobre a frequência com que utilizam essa ferramenta.

A terceira parte do questionário visou criar uma escala do uso de tecnologias pelos integrantes da Geração Y. Após a conclusão da pesquisa percebeu-se que a frequência de uso das tecnologias é grande, porém são sempre as mesmas, não havendo grande diversidade. As mais usadas envolvem as comunicações rápidas e as pesquisas na internet, englobando: uso das redes sociais para checar atualizações, comentar publicações, receber e/ou enviar mensagens e chamadas no celular, pesquisas específicas, assistir vídeos e/ou escutar música online. Algumas das tecnologias citadas pelo seu potencial educacional, como sites e blogs (Ferdig \& Trammell, 2004), ou por ter grande adesão dos integrantes da Geração Y, como jogos (Prensky, 2009), não obtiveram respostas que indicassem alta frequência de uso pelos participantes do estudo, essa contradição apresenta-se também em alguns estudos realizados tanto na Austrália (Kennedy, Dalgarno \& Waycott, 2010) como no Canadá (Guo, Dobson \& Petrina, 2008).

\section{Conclusão}

Esse estudo analisou as características atribuídas à Geração Y, avaliando o perfil digital dos estudantes, seus hábitos eficazes de aprendizagem, as tecnologias usadas e as suas complexas relações. A conclusão obtida é que os alunos não usam uma grande variedade de tecnologias e as que são utilizadas não atingem seu aproveitamento máximo, no contexto da educação.

Esses mesmo autores afirmam que os nativos digitais conseguiriam ser autossuficientes em sua aprendizagem se possuíssem as tecnologias necessárias para pesquisas, contudo esse estudo mostrou que os professores têm um papel crucial em prepara-los para utilizar as ferramentas digitais no aprendizado. Por exemplo, eles podem ajudar os estudantes a desenvolverem habilidades para utilizar ferramentas de pesquisas, como o Pubmed, ou ainda a desenvolverem estratégias para evitar distrações que podem ser causadas pelo uso das tecnologias durante os estudos.

O estudo permitiu concluir ainda que os alunos são favoráveis tanto a aulas (forma indireta) como a realização de projetos (forma direta), mostrando que compreendem a necessidade da existência das duas formas de aquisição de conhecimento; consideram o tédio de curto prazo gerado pelas aulas como necessário e, portanto, tentam se manter atentos. A tarefa 
do professor é, pois, amenizar a dispersão durante as aulas, interagindo com os alunos e ajudando-os a navegar pelas novas tecnologias e aproveitá-las da melhor forma possível.

A tecnologia exerce importante influência na vida dos estudantes, porém continua sendo uma entre várias outras, o que faz com que o professor mantenha um papel importantíssimo na formação desses alunos considerados nativos digitais, principalmente ajudando e os guiando-os na utilização correta e completa das novas tecnologias desenvolvidas para os estudos.

\section{Referências}

Bauerlein, M., \& Walesh, S. G. (2009). The Dumbest Generation-How the Digital Age Stupefies Young Americans and Jeopardizes Our Future. Jeremy P. Tarcher/Penguin, New York, 2008. VISUALIZAR ITEM GS SEARCH

Cain, J., \& Policastri, A. (2011). Using Facebook as an informal learning environment. American journal of pharmaceutical education, 75(10), 207. VISUALIZAR $\underline{\text { ITEM GS SEARCH }}$

Castanha, D., \& Castro, M. B. (2010). A necessidade de refletir sobre as estratégias pedagógicas para atender à aprendizagem da Geração Y. Revista de EDUCAÇÃO do Cogeime, 19(36), 27-38. VISUALIZAR ITEM GS SEARCH

Del Prette, A., \& Del Prette, Z. A. P. (2003). No contexto da travessia para o ambiente de trabalho: treinamento de habilidades sociais com universitários. Estudos de psicologia, 8(3), 413-420. VISUALIZAR ITEM GS SEARCH

Ebner, M., Nagler, W., \& Schön, M. (2012, June). Have they changed? Five years of survey on academic net-generation. In Proceedings of World Conference on Educational Multimedia, Hypermedia and Telecommunications (pp. 343-353). VISUALIZAR ITEM GS SEARCH

Ferdig, R. E., \& Trammell, K. D. (2004). Content delivery in the'Blogosphere'. THE Journal (Technological Horizons In Education), 31(7), 12. VISUALIZAR ITEM GS SEARCH

Guo, R. X., Dobson, T., \& Petrina, S. (2008). Digital natives, digital immigrants: An analysis of age and ICT competency in teacher education. Journal of educational computing research, 38(3), 235-254. VISUALIZAR ITEM GS SEARCH

Jones, C. (2012). Networked learning, stepping beyond the net generation and digital natives. In Exploring the theory, pedagogy and practice of networked learning (pp. 27-41). Springer New York. VISUALIZAR ITEM GS SEARCH

Jones, C., Ramanau, R., Cross, S., \& Healing, G. (2010). Net generation or Digital Natives: Is there a distinct new generation entering university? Computers \& Education, 54(3), 722732. VISUALIZAR ITEM GS SEARCH

Jimoyiannis, A., \& Angelaina, S. (2012). Towards an analysis framework for investigating students' engagement and learning in educational blogs. Journal of Computer Assisted Learning, 28(3), 222-234. VISUALIZAR ITEM GS SEARCH

Kennedy, G., Judd, T., Dalgarno, B., \& Waycott, J. (2010). Beyond natives and immigrants: exploring types of net generation students. Journal of Computer Assisted Learning, 26(5), 332-343. VISUALIZAR ITEM GS SEARCH

Kennedy, G. E., \& Judd, T. S. (2011). Beyond Google and the "satisficing" searching of digital natives. Deconstructing digital natives, 119-136. VISUALIZAR ITEM GS SEARCH

Kenski, V. M. (2003). Aprendizagem mediada pela tecnologia. Revista diálogo educacional, Curitiba, 4(10), 47-56. VISUALIZAR ITEM GS SEARCH 
Lombardía, P. G. (2008). Quem é a geração Y? HSM Management, (n.70), p.1-7. Retrieved from http://www.hsm.com.br/artigos/quem-e-geracao-y VISUALIZAR ITEM

Oliveira, S. (2015). Encontro de gerações: geração Y. VISUALIZAR ITEM GS SEARCH

Prensky, M. (2009). H. sapiens digital: From digital immigrants and digital natives to digital wisdom. Innovate: journal of online education, 5(3), 1. VISUALIZAR ITEM $\underline{\text { GS }}$ $\underline{\text { SEARCH }}$

Prober, C. G. (2012). Lecture halls without lectures--a proposal for medical education. The New England journal of medicine, 366(18), 1657. VISUALIZAR ITEM

Prodanov, C. C., \& Freitas, E. C. (2013). Metodologia do Trabalho Científico: Métodos e Técnicas da Pesquisa e do Trabalho Acadêmico-2 ${ }^{a}$ Edição. Editora Feevale. VISUALIZAR ITEM GS SEARCH

Rennie, F., \& Morrison, T. (2013). E-learning and social networking handbook: Resources for higher education. Routledge. VISUALIZAR ITEM GS SEARCH

Santos, T. B. B. dos, \& Carvalho, M. C. A. de. (2009). Vídeo com modelos esquemáticos descrevendo a formação do intestino faríngeo. Retrieved May 7, 2013, from http://videoaulas.uff.br/conteudo/embriologia-intestino-far?ngeo VISUALIZAR ITEM

Simões, L., \& Gouveia, L. (2008). Geração Net, Web 2.0 e ensino superior. Cadernos de Estudos Mediáticos, (6), 21-32. VISUALIZAR ITEM GS SEARCH

Thompson, P. (2013). The digital natives as learners: Technology use patterns and approaches to learning. Computers \& Education, 65, 12-33. VISUALIZAR ITEM GS SEARCH

Torres, T. Z., \& Amaral, S. F. (2011). Aprendizagem Colaborativa e Web 2.0: proposta de modelo de organização de conteúdos interativos. Educação Temática Digital, 12, 49. VISUALIZAR ITEM GS SEARCH

Twenge, J. M. (2009). Generational changes and their impact in the classroom: teaching Generation Me. Medical education, 43(5), 398-405. VISUALIZAR ITEM GS SEARCH

USP. (2016). Projeto Homem Virtual. Retrieved August 7, 2016, from http://telemedicina.fm.usp.br/portal/projeto-homem-virtual/ $\underline{\text { VISUALIZAR ITEM GS }}$ $\underline{\text { SEARCH}}$ 